\title{
The Contribution of Fires to TES Observations of Free Tropospheric PAN over North America in July
}

Emily V. Fischer ${ }^{1}$, Liye Zhu ${ }^{1}$, Vivienne H. Payne ${ }^{2}$, John R. Worden ${ }^{2}$, Zhe Jiang ${ }^{4}$, Susan S. Kulawik ${ }^{3}$, Steven Brey ${ }^{1}$, Arsineh Hecobian ${ }^{1}$, Daniel Gombos ${ }^{7}$, Karen Cady-Pereira ${ }^{5}$, and Frank Flocke ${ }^{6}$

${ }^{1}$ Department of Atmospheric Science, Colorado State University, Fort Collins, CO, USA

${ }^{2}$ Jet Propulsion Laboratory, California Institute of Technology, Pasadena, CA, USA

${ }^{3}$ Bay Area Environmental Research Institute Moffett Field, Moffett Field, CA, USA

${ }^{4}$ National Center for Atmospheric Research, Boulder, CO, USA

${ }^{5}$ Atmospheric and Environmental Research (AER), Lexington, MA, USA

${ }^{6}$ National Center for Atmospheric Research (NCAR), Boulder, CO, USA

${ }^{7}$ MORSE Corp, Cambridge, MA, USA

Correspondence to: Emily V. Fischer (evf@atmos.colostate.edu)
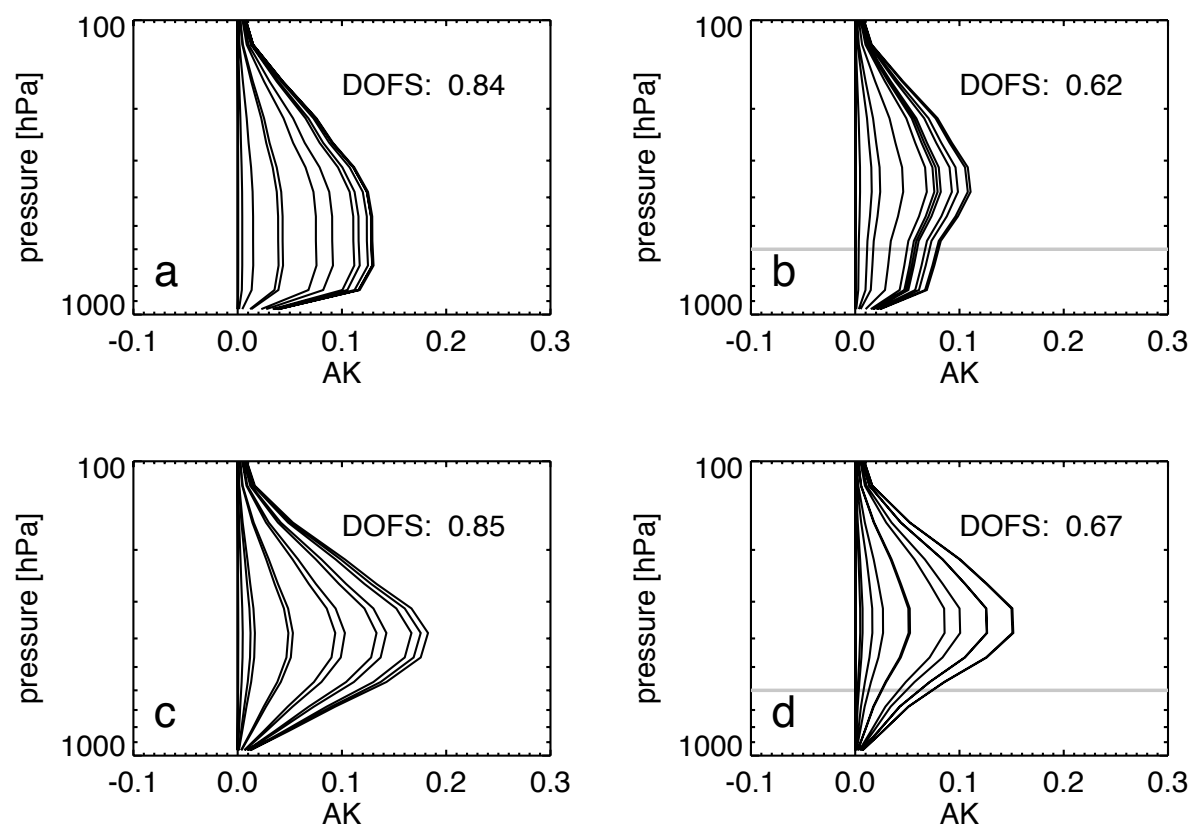

Figure S1: Averaging Kernels (AK) associated with the simulated TES PAN retrievals for four different hypothetical conditions shown in Figure 1. 

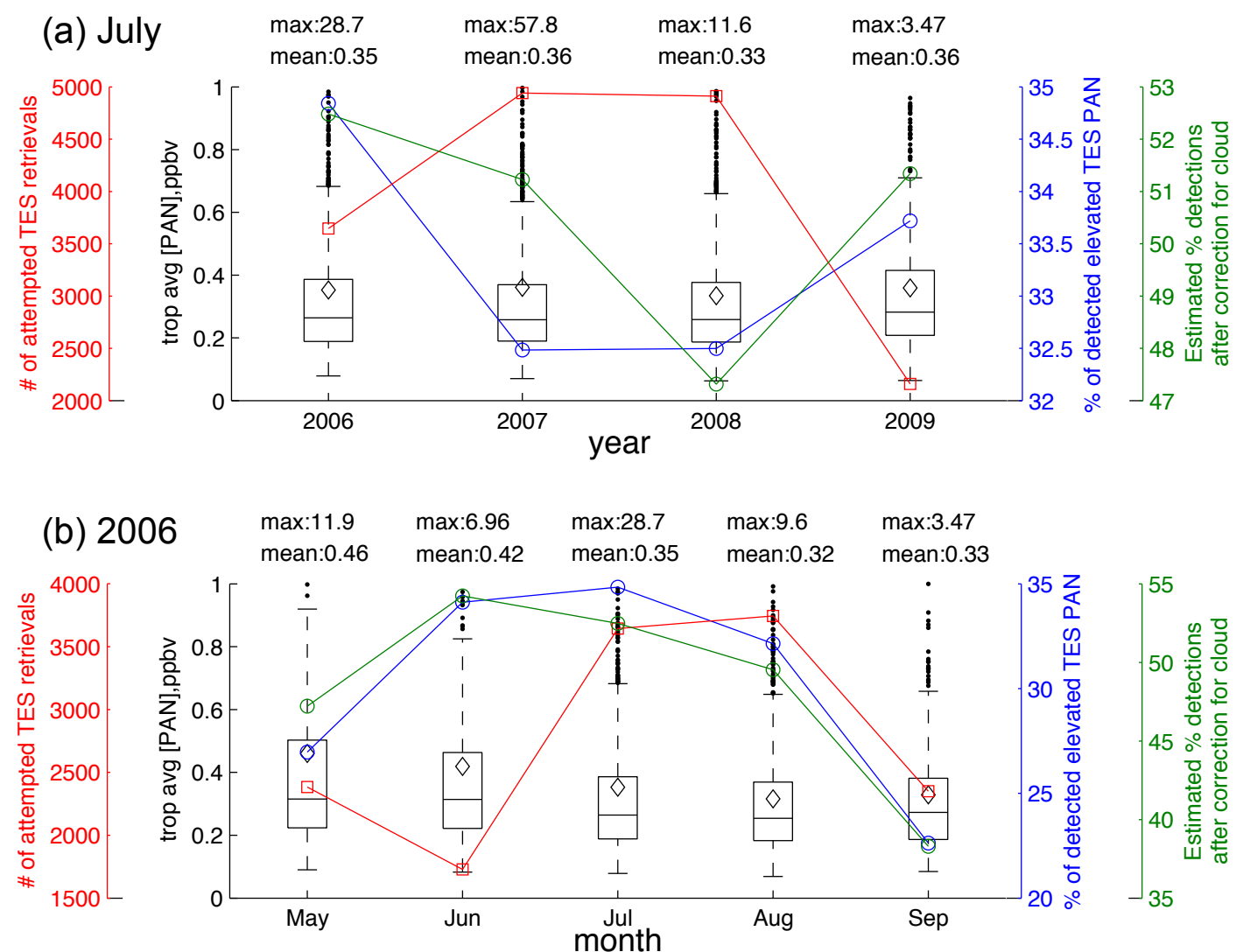

Figure S2: Summary statistics of the variability of free tropospheric average TES PAN over North America between May and September 2006 (top panel) and July 2006 to 2009 (bottom panel). The information presented here includes the number of attempted TES PAN retrievals over the eastern Pacific Ocean (red), the percentage of observations where elevated PAN was detected (blue), the percentage of successful TES PAN detections corrected for the presence of clouds (green), and the monthly distributions (black box plots) for the free tropospheric average PAN. The green line was calculated by dividing the percentage of successful detections (blue line) by the percentage of total attempted retrievals with cloud optical depths $<0.5$. The boxes enclose the $25^{\text {th }}$ to $75^{\text {th }}$ percentiles, the whiskers represent the $5^{\text {th }}$ and $95^{\text {th }}$ percentiles, the black dots are the outliers, the horizontal bar represents the median, and the diamond represents the mean. The max and mean values of each year are on the top of the boxes. As throughout the paper, we define North America to include the contiguous U.S. $\left(125^{\circ} \mathrm{W}-70^{\circ} \mathrm{W}, 30^{\circ} \mathrm{N}-50^{\circ} \mathrm{N}\right)$ and southern Canada $\left(130^{\circ} \mathrm{W}-60^{\circ} \mathrm{W}, 50^{\circ} \mathrm{N}-70^{\circ} \mathrm{N}\right)$. 


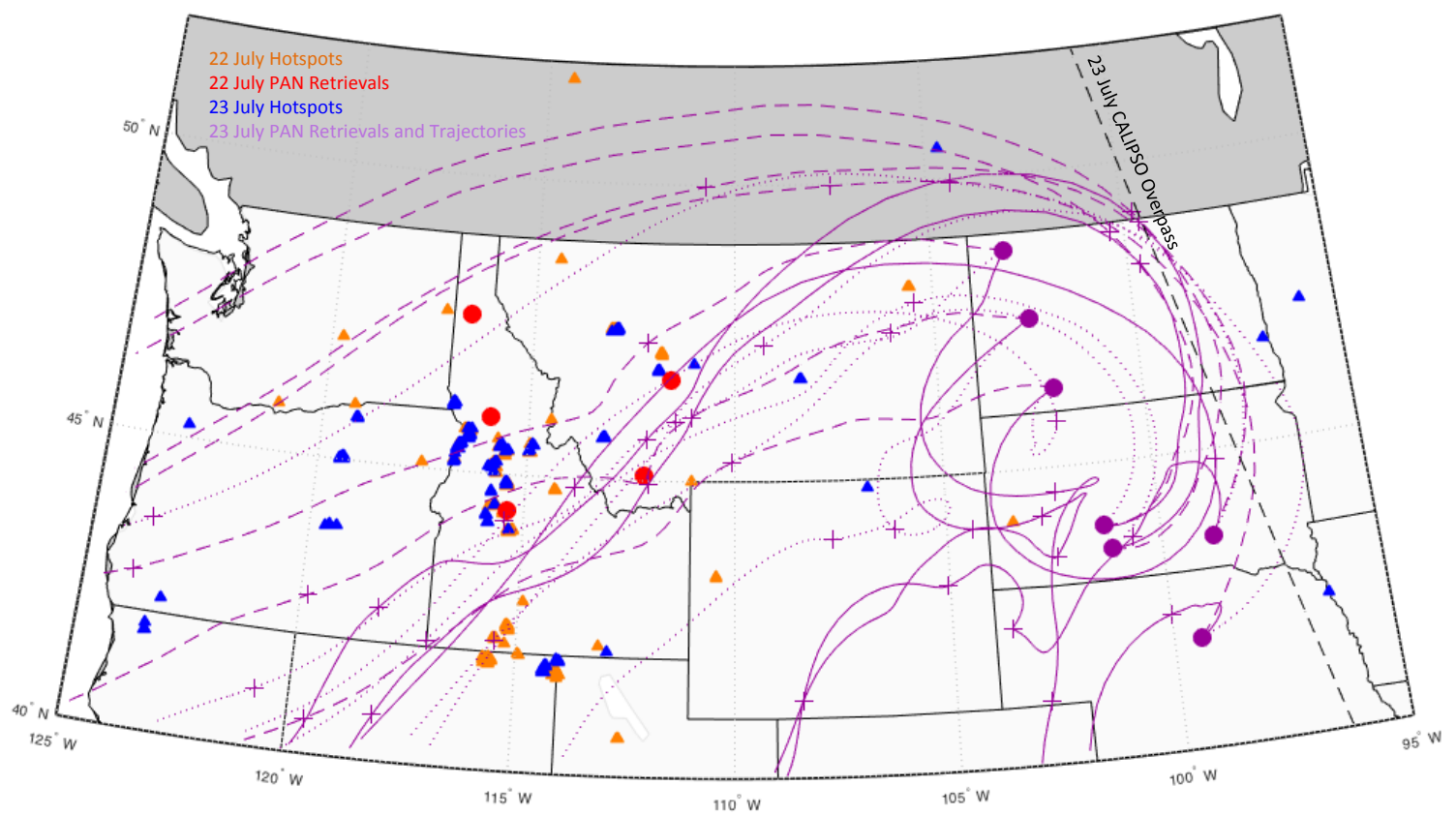

Figure S3: Case study of TES PAN retrievals overlapping HMS smoke polygons 22 - 23 July 2007.

Orange triangles represent FIRMS MODIS Hotspots for 22 July (Product MCD14ML;

https://firms.modaps.eosdis.nasa.gov/download/request.php). Blue triangles represent FIRMS MODIS

Hotspots for 23 July. Red circles indicate TES PAN retrievals on 22 July, and purple circles represent TES

PAN retrievals on 23 July. The purple lines signify 5 day HYSPLIT backward trajectories initialized at each TES retrieval at $2 \mathrm{~km}$ (solid lines), $4 \mathrm{~km}$ (dotted lines), and $6 \mathrm{~km}$ (dashed lines). The purple '+' signifies 24 hours of transport time on the $4 \mathrm{~km}$ trajectories. 


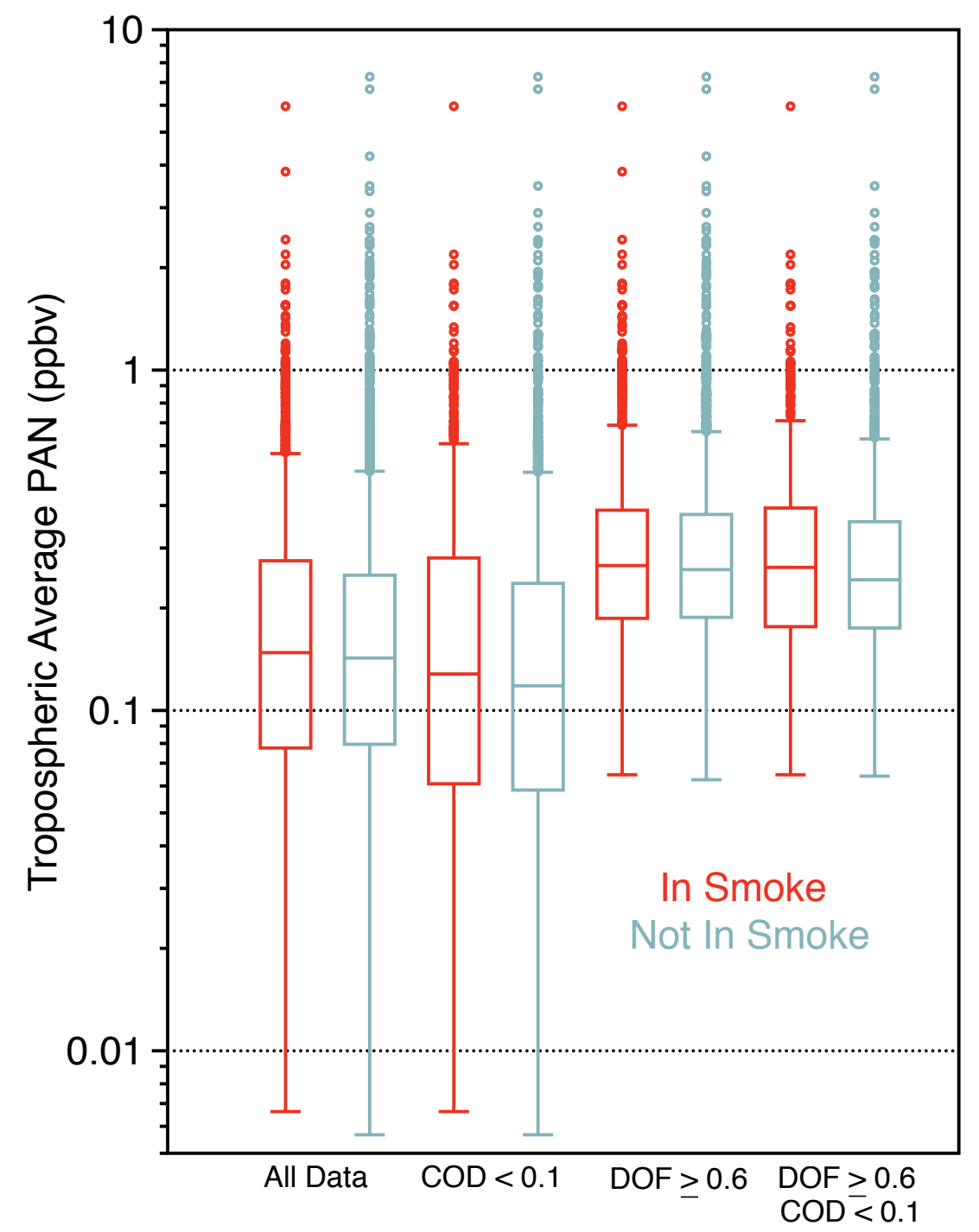

Figure S4: Box plots of July 2006 - 2009 North American TES PAN retrievals overlapping HMS smoke plume polygons ("In Smoke"; red), and TES PAN retrievals not overlapping HMS smoke plume polygons ("Not In Smoke"; blue-grey). The first set of box plots is for all TES PAN retrievals. The second set of box plots includes a subset of this data where cloud optical depth (COD) is $<0.1$. The third set of box plots includes all data with DOF $>0.6$. The final (rightmost) set of box plots includes all data with DOF $>0.6$ and $\mathrm{COD}<0.1$. The box plots display the interquartile range for each subset and the dots represent outliers. 